\title{
Ventilator-associated pneumonia related to ESBL-producing gram negative bacilli
}

\author{
Benoit Pilmis ${ }^{1}$, Jean-Ralph Zahar ${ }^{2,3}$ \\ ${ }^{1}$ Antimicrobial Stewardship Team, Hospital Paris Saint Joseph, 185 rue Raymond Losserand, 75014 Paris, France; ${ }^{2}$ Department of Microbiology, \\ Hospital Paris Seine Saint Denis, 125 rue de Stalingrad 93000 Bobigny, France; ${ }^{3}$ UMR 1137, IAME Team 5, DeSCID: Decision SCiences in \\ Infectious Diseases, Control and Care, INSERM, UFR SMBH, Paris 13, Paris Cité University, Paris, France \\ Contributions: (I) Conception and design: JR Zahar; (II) Administrative support: None; (III) Provision of study materials or patients: B Pilmis; (IV) \\ Collection and assembly of data: B Pilmis; (V) Data analysis and interpretation: None; (VI) Manuscript writing: All authors; (VII) Final approval of \\ manuscript: All authors. \\ Correspondence to: Jean-Ralph Zahar. Infection Control Unit- Groupe Hospitalier Paris Seine Saint-Denis, 125 rue de Stalingrad 93000 Bobigny, \\ France. Email: jeanralph.zahar@aphp.fr.
}

\begin{abstract}
Ventilator-associated pneumonia (VAP) is one of the most frequent cause of intensive care unit (ICU) acquired infections. The worldwide spreading of extended spectrum beta-lactamase producing enterobacteriaceae (ESBL-PE) represents a major problem encountered more and more frequently in ICU. Among ICU patients, between $5 \%$ to $25 \%$ are ESBL-PE carriers. Whereas, previous carriage is the major risk factors associated with VAP related to ESBL-PE, among carriers, only 5\% to $20 \%$ will develop a VAP related to ESBL-PE. Also, diagnosis and therapeutic delay are associated with length of stay and higher morbidity, and mortality, therefore, early identification of patients at risk of ESBL-PE related infections is crucial for early implementation of effective antibiotic therapy. VAP related to ESBL-PE should be considered in: previous colonized patients in case of late onset pneumonia and/or when several antibiotic courses precede the infectious episode or even in patients with shock. Among non-colonized patients, if VAP occurs, the risk being related to ESBL-PE is less than $1 \%$. In the future, new rapid microbiological diagnostic tests will allow an early diagnosis. According to recent data, empirical antibiotic therapy should be based on carbapenems. Other alternative antibiotic classes could be used for de-escalation. However, several pharmacodynamic and pharmacokinetics precautions should be taken to achieve drug concentrations at site of infection and except to cure the infected patient.
\end{abstract}

Keywords: Ventilator-associated pneumonia (VAP); extended spectrum beta-lactamase (ESBL); carbapenem; colonization

Submitted Jul 18, 2018. Accepted for publication Aug 23, 2018.

doi: $10.21037 /$ atm.2018.09.34

View this article at: http://dx.doi.org/10.21037/atm.2018.09.34

\section{Introduction}

Ventilator-associated pneumonia (VAP) is defined as pneumonia occurring among mechanically ventilated patients for at least 48 hours (1). VAP is associated with a high morbidity and mortality. It is one of the most common nosocomial infections acquired in intensive care unit (ICU), and a frequent cause of mortality $(2,3)$. VAP incidence ranges from 2 to 16 episodes per 1,000 ventilator-days and it increase duration of ICU and inhospital stay (3). Furthermore the risk of VAP occurring increased every day by $1.5 \%$ per day. The most frequent isolated microorganisms are Pseudomonas aeruginosa and Staphylococcus aureus. However, previous antibiotic therapy and delay of VAP occurrence are the main factors associated with involvement of multidrug resistant organisms. Enterobacteriaceae are frequently isolated and Escherichia coli and Klebsiella species reach $20 \%$ of all isolated bacteria. 
Extended spectrum beta lactamase producing enterobacteriaceae (ESBL-PE) are increasing worldwide since 2000. In Europe and North-America, authors estimate up to $10 \%$ of community populations are colonized whereas, in low-income countries colonization reached in the community up to $40 \%$ (4). The successful spread of ESBL-PE in healthcare settings and the community is associated with a higher risk of infection related to these pathogens. ICU patients are at particular risk of infection, as ESBL-PE reached critical levels in this specific population. Indeed, several recent studies suggested that, ESBL-PE colonization was identified among 5\% to 30\% of ICU-admitted patients. In the recent annual report of ECDC-Europa 32\% among Enterobacteriaceae isolated from pneumonia were resistant to third generation cephalosporins and the most frequent involved species were Escherichia coli and Klebsiella pneumoniae.

The rate of colonization in a different population depend of several factors. Indeed, the risk increase according to the local prevalence, and it seems more common in the South Asian, populations compared to Mediterranean and African countries. Also, there is a risk gradient in Europe, with a high prevalence in southern compared to northern countries. The local prevalence reflects the community spreading, however, other well-known risk factor had been published. Indeed, some specific populations, because of several hospitalizations and antibiotic courses, are exposed to a higher risk of rectal carriage as kidney and liver transplant patients. Also, specific risk factors had been published, as previous hospitalization, recurrent urinary tract infection, age older than 65 , previous antibiotic therapy and particularly third generation cephalosporins and fluoroquinolones. Recent studies suggested that traveling abroad was associated with a higher risk of acquisition, also the risk seems to be associated with the visited countries (more frequent for South Asian country than Mediterranean). However, the duration of carriage remains unknown (5). Other risk factors have been reported such as co-morbidities, hospitalisation in ICU, length of stay in ICU prior to infection.

\section{ESBL-PE carriage and risk of infection in ICU}

In a recent study conducted in France, authors suggested that, at ICU-admission, among all admitted patients, $15 \%$ were ESBL-PE carriers $(6,7)$. However, ESBL-PE carriage reached $22 \%$ of patients admitted from the hospital. Also, ESBL-PE digestive colonization increased with the duration of ICU stay, and was as frequent as $15.6 \%$ for stays shorter than 5 days to $37 \%$ for longer stay (8).

The increased prevalence of carriage is associated with a higher risk of secondary ESBL-PE related infection in patients. Carriage being the main prerequisite for acquired ESBL-PE related infections (4). While prior ESBL$\mathrm{PE}$ colonization is the major risk factor for ESBL-PE infections, several studies, conducted in and out of ICUs, suggested that among previously known colonized patients, less than $10 \%$ will develop an infection (9). Current guidelines, suggest to consider the colonization statutes in order to adapt the empirical antibiotic choices in case of ICU-acquired infection (10). However, this attitude results in excess consumption of broad-spectrum antibiotics, leading to the dissemination of pandrug resistant organisms. Also, according to these data, it seems crucial to identify among ESBL-PE carriers with ICU-acquired infections those that are prone to be infected with an ESBL-PE.

\section{Risk of infections, among ESBL-PE carriers}

Several studies investigated, the risk factors associated with ESBL-PE related infections whether it as community or healthcare acquired infections. Two main risk factors seem associated with ESBL-PE related infections: previous antibiotic therapy and invasive devices.

In a prospective cohort study conducted in 50 French spoken hospital (11), authors identified three risk factors associated with nosocomial ESBL-PE related BSI. In this study, travel to high-risk country within the last 3 years, high regional incidence and previous anti anaerobic antibiotic administration were significantly associated with nosocomial ESBL-PE related BSI. Rodriguez-Bano, performed a retrospective case control study in 13 Spanish hospitals to investigate risk factors associated with ESBLEscherichia coli (ESBL-EC) bloodstream infections (BSI). In this study, authors identified 3 risk factors and one protective factor associated with bloodstream infections. Duration of stay before infection, organ transplantation (odds and previous antibiotic therapy (use of oxyimino-beta-lactams) were associated by multivariate analysis with the occurrence of bloodstream infection related to ESBL-PE. Whereas unknown BSI source was identified as protective (12).

A recent study with the aim to validate a decision tree for prediction ESBL-PE infection highlighted five factors associated with the occurrence of a ESBL-PE related infection. These factors were: (I) a history of ESBL-PE colonization/infection, (II) age $\geq 43$ years, (III) 
recent hospitalization in an ESBL high-burden region, (IV) $\geq 6$ days of antibiotic exposure in the prior 6 months and $(\mathrm{V})$ chronic indwelling vascular hardware. The positive and negative predictive values of this algorithm were $90.8 \%$ and $91.9 \%$ (13). Furthermore, a recent retrospective case-control study conducted in the United States by Augustine et al. (14) suggested that $5 \%$ among BSI were related to ESBL-PE. In this study of 910 patients with enterobacteriaceae BSI, most of BSI related to ESBL-PE were community onset (79\%). Also 60\% were related to $E$. coli. Authors identified 3 risk factors associated with ESBL$\mathrm{PE}$ bloodstream infections. Indeed, (I) procedures within 1 month (aOR: 8.7, 95\% CI: 3.1-22.9), (II) prior infections or colonization with ESBL-PE within 12 months (aOR: $26.8,95 \%$ CI: $7.0-108.2$ ), and (III) number of prior courses of $\beta$-lactams and/or fluoroquinolones within 3 months before BSI: 1 course (aOR: 6.3, $95 \%$ CI: 2.7-14.7), 2 courses (aOR: 22.0, 95\% CI: 8.6-57).

Few studies are interested to investigate the risk factors associated in previous colonized patients. In a retrospective case-controlled study, conducted out of ICU and including pediatric and adult patients, authors identified 2 factors, associated with ESBL-Escherichia coli infection in previously colonized patients. These 2 factors were the use of $\beta$-lactam/ $\beta$-lactamase inhibitor prior to infection and urinary catheterization were the only to risk factors identified (9).

More recently, a Sweden group, followed subjects with ESBL-PE isolated from rectal or urine samples during a 6-year period. They assessed the risk of BSI during the period of follow-up. The main results of this populationbased cohort study were: firstly, the cumulative 6-year BSI incidences were less than $4 \%$ and secondly the incidences decreased during the first year of follow-up. In this study, urological disorders, were associated with the higher risk of infection. Also, several antibiotics without activity against ESBL-PE were associated with a higher risk of infection in previous colonized patients, within the 3 months after antibiotic dispensation (15). In ICU the risk of EBLSE-PE related infection seems to be rare, including in previously colonized patients (6). In a first published study conducted in France, among previously known carriers, ESBLPE caused $10 \%$ and $27 \%$ of first and second episodes of ICU-acquired infections, respectively. Recently, the same authors, assessed the prevalence and associated factors of ESBL-PE pneumonia acquired in ICU among carriers. In a prospective study conducted between 2009 and 2015, authors identified 13\% ESBL-PE carriers. Among them $13 \%$ developed ICU-acquired pneumonia of whom $43 \%$ were due to ESBL-PE. Also this represented only 6\% among of carriers (16). By multivariable analysis, among previously colonized patients, predictive factors for ESBL$\mathrm{PE}$ related pneumonia 2 risk factors were associated with a higher risk of infection: SAPS II at admission and colonization with non-E.coli species (Enterobacter sp. or $K$. pneumoniae species)In this study a protective factor was identified. Indeed, prior administration of amoxicillinclavulanic acid for more than 48 hours was associated with a lower risk of infection. Two other studies conducted in ICU underlined the fact that ESBL-BE represented less than $1 \%$ among all infected patients $(17,18)$. These two studies suggested a positive and negative values of $41.5 \%$ and 99.4\%, respectively, for ESBL-PE carriage for predicting ESBL-PE related. In another study authors conducted a multicentric retrospective observational study, including every patient with known status regarding ESBL-PE rectal colonization and a respiratory tract specimen positive culture. This study included 1,503 patients representing 2,498 respiratory tract samples, $1,557(62.3 \%)$ respiratory samples were performed early within the first 5 days of ICU stay and 941 (37.7\%) later than 5 days. Positivity rates for ESBL-E were $15 \%$ and $37 \%$ for rectal swabs in the early and late groups, respectively. Sensitivity, specificity, positive (PPV) and negative (NPV) predictive values and likelihood ratios were calculated for ESBL-E digestive colonization as a predictor of ESBL-E presence in respiratory samples. PPVs of ESBL-E digestive colonization were 14.5\% (95\% CI: $12.8-16.3$ ) and $34.4 \%$ (95\% CI: 31.4-37.4), for the early and late groups, respectively, whereas NPVs were $99.2 \%$ (95\% CI: 98.7-99.6) and 93.4\% (95\% CI: 91.9-95.0), respectively (8). Finally, a recent paper aim to investigate, among ESBL-PE colonized patients, the significance of infection-related ventilator-associated complications (IVAC) in mechanically ventilated patients. Authors highlighted, among 318 previous ESBL-PE colonized patients, 21\% IVAC related to infections other than VAP, $13 \%$ related to non-ESBL-PE VAP and 3\% related to ESBL-PE VAP. In this retrospective study, ESBL-PE related infections accounted for only $7 \%$ episodes. Also, by multivariate analysis, one factor was associated with a protective effect. Indeed, carbapenem given within the 3 days before the IVAC episode reduce significantly the risk (18).

\section{Which is the best available beta-lactams for ESBL-PE related VAP?}

Therapy of invasive infections due to ESBL-PE is 
challenging, as the only class antibiotic that seems to be constantly effective are carbapenems. MDRO carriage is frequently associated with a higher consumption of late resort antibiotics even in the absence of infection (19). Two recent study conducted in (19) and out of ICU (20) suggested that ESBL-PE carriage also is associated with higher carbapenem exposure than in non-carriers. Using carbapenem to all suspected pneumonia in ESBL-PE colonized patients will expose to an over consumption of this last resort antibiotic, which will increase the risk of emerging and spreading of resistant isolates to carbapenems. Indeed prior therapy with carbapenem had been previously associated as a risk factors of (I) intestinal carriage of imipenem-resistant GNB (IR-GNB) in intensive care patients, almost exclusively Pseudomonas aeruginosa (21) and (II) with both carbapenem and beta-lactam resistance in endemic $P$. aeruginosa (22). According to these data, several authors suggested the use of "alternative" antibiotics to carbapenems to treat ESBL-PE related infections (23). Indeed, several studies suggested that ESBL-PE were susceptible to non-carbapenem beta-lactams. However, the ecological value of saving carbapenems had never been clearly demonstrated. Numerous clinical studies have documented the emergence of carbapenem producing enterobacteriaceae in association with the use of various antibiotic treatment (24).

The susceptibility of ESBL-PE to non-carbapenems antibiotic classes depends on several factors as: the species concerned, the antibiotic class tested, and local epidemiology. ESBL-producing E. coli is usually regarded as more susceptible to all beta-lactams than ESBL-producing $K$. pneumoniae, and non-E. coli species. Piperacillin-tazobactam (Pip-Taz) being the most effective antibiotic (25) compared to third and fourth generation cephalosporins. However, treatment with such antibiotics had been associated with high failure rates and should be questioned in ICU infected patients. This failure rate could be explained by the difficulty in achieving the PK/PD parameters necessary for the effectiveness of antibiotic therapy $(26,27)$. That's why for ICU patients the in vitro susceptibility tests can not be the sole criterion for using an antibiotic. Several other criteria should be taken into account due to increase of distribution volume and renal clearance in case of severe infection. Also the probability of attaining therapeutic drug levels in ICU patients is the first step after antibiotic susceptibility test to allow or not the use of «alternatives» to carbapenems.
Recent pharmacological studies using pharmacokinetics modelisation suggested for ESBL-PE infection, the need to increase the doses administered and prolonged infusion when using piperacillin-tazobactam for treating ESBL-PE infections (28).

Several other beta-lactams had been proposed to treat ESBL-PE related infection in ICU patients as, Cefoxitin (cephamycin a second-generation cephalosporin), temocillin (a stable antibiotic against hydrolysis by ESBLs and Ampc beta-lactamases), beta lactams-beta-lactamase inhibitor combinations such as piperacillin-tazobactam or the new ones (ceftolozane-tazobactam or ceftazidime-avibactam). With the exception of carbapenems none of the antibiotics mentioned is constantly active on ESBL-PE. Also this must have led us to use these antibiotics in a reasonable way and certainly not empirically. Indeed, when practitioner suspected an ESBL-PE related acquired pneumonia, before obtaining the result of antibiotic susceptibility test, the only antibiotic that could be used are carbapenems. Also, all the other ones should be discussed, at time of de-escalation when antibiotic susceptibility tests are available, and the clinical situation is improved. All the antibiotics mentioned above could be used, if, firstly the ESBL-PE isolate is susceptible in-vitro and, and secondly needs adapting doses and modes of administration in order to reach the required concentrations especially in the most serious patients.

It seems that the use of carbapenems is recognized by all actors as associated with major ecological consequences. However, recent data suggested that imipenem had no global effect on microbiota. Indeed a recent limited study conducted on 17 patients receiving imipenem, showed by metagenomic techniques, unexpectedly limited effects on microbiota, possibly because of very low imipenem intestinal concentrations (29). However, in an era of pandrug resistant organisms, it seems important to better identify among the colonize population those that need as firstline antibiotic therapy the antibiotic with a broad spectrum.

To resolve this question, rapid diagnostic tests had been recently developed. Chromogenic tests as the «beta Lacta test had been evaluated on isolate culture and recently on bronchial aspirate samples (30). In this recent study beta Lacta Test detected ESBL-PE-GNB directly on clinical BAS positive for GNB on microscopic Gram staining examination and/or growing with $\geq 10^{4} \mathrm{CFU} / \mathrm{mL}$ with $100 \%$ sensitivity, specificity, and positive and negative predictive values. BLT seems to be an accurate tool for ESBL-PEGNB detection directly on BAS. 


\section{Conclusions}

ESBL-producing enterobacteriaceae related VAP remains a rare event in intensive care units, especially in patients without prior known colonization. The occurrence of these infections seems to be favored by previous antibiotic therapy. However, in patients with septic shock and anterior colonization with ESBL-producing enterobacteriaceae, carbapenems remain the reference treatment. New microbiological methods for rapid identification of resistance mechanisms look promising and deserve future studies.

\section{Acknowledgements}

None.

\section{Footnote}

Conflicts of Interest: The authors have no conflicts of interest to declare.

\section{References}

1. Timsit JF, Esaied W, Neuville M, et al. Update on ventilator-associated pneumonia. F1000Res 2017;6:2061.

2. Torres A, Niederman MS, Chastre J, et al. International ERS/ESICM/ESCMID/ALAT guidelines for the management of hospital-acquired pneumonia and ventilator-associated pneumonia: Guidelines for the management of hospital-acquired pneumonia (HAP)/ ventilator-associated pneumonia (VAP) of the European Respiratory Society (ERS), European Society of Intensive Care Medicine (ESICM), European Society of Clinical Microbiology and Infectious Diseases (ESCMID) and Asociación Latinoamericana del Tórax (ALAT). Eur Respir J 2017;50.

3. Rosenthal VD, Bijie H, Maki DG, et al. International Nosocomial Infection Control Consortium (INICC) report, data summary of 36 countries, for 2004-2009. Am J Infect Control 2012;40:396-407.

4. Detsis M, Karanika S, Mylonakis E. ICU Acquisition Rate, Risk Factors, and Clinical Significance of Digestive Tract Colonization With Extended-Spectrum Beta-LactamaseProducing Enterobacteriaceae: A Systematic Review and Meta-Analysis. Crit Care Med 2017;45:705-14.

5. Ruppé E, Armand-Lefèvre L, Estellat C, et al. High Rate of Acquisition but Short Duration of Carriage of Multidrug-Resistant Enterobacteriaceae After Travel to the Tropics. Clin Infect Dis 2015;61:593-600.

6. Razazi K, Derde LPG, Verachten M, et al. Clinical impact and risk factors for colonization with extended-spectrum $\beta$-lactamase-producing bacteria in the intensive care unit. Intensive Care Med 2012;38:1769-78.

7. Pilmis B, Cattoir V, Lecointe D, et al. Carriage of ESBLproducing Enterobacteriaceae in French hospitals: the PORTABLSE study. J Hosp Infect 2018;98:247-52.

8. Carbonne H, Le Dorze M, Bourrel AS, et al. Relation between presence of extended-spectrum $\beta$-lactamaseproducing Enterobacteriaceae in systematic rectal swabs and respiratory tract specimens in ICU patients. Ann Intensive Care 2017;7:13.

9. Goulenok T, Ferroni A, Bille E, et al. Risk factors for developing ESBL E. coli: can clinicians predict infection in patients with prior colonization? J Hosp Infect 2013;84:294-9.

10. Bretonnière $\mathrm{C}$, Leone $\mathrm{M}$, Milési $\mathrm{C}$, et al. Strategies to reduce curative antibiotic therapy in intensive care units (adult and paediatric). Intensive Care Med 2015;41:1181-96.

11. Zahar JR, Lesprit P, Ruckly S, et al. Predominance of healthcare-associated cases among episodes of communityonset bacteraemia due to extended-spectrum $\beta$-lactamaseproducing Enterobacteriaceae. Int J Antimicrob Agents 2017;49:67-73.

12. Rodríguez-Baño J, Navarro MD, Retamar P, et al. $\beta$-Lactam/ $\beta$-lactam inhibitor combinations for the treatment of bacteremia due to extended-spectrum $\beta$-lactamase-producing Escherichia coli: a post hoc analysis of prospective cohorts. Clin Infect Dis 2012;54:167-74.

13. Goodman KE, Lessler J, Cosgrove SE, et al. A Clinical Decision Tree to Predict Whether a Bacteremic Patient Is Infected With an Extended-Spectrum $\beta$-LactamaseProducing Organism. Clin Infect Dis 2016;63:896-903.

14. Augustine MR, Testerman TL, Justo JA, et al. Clinical Risk Score for Prediction of Extended-Spectrum $\beta$-LactamaseProducing Enterobacteriaceae in Bloodstream Isolates. Infect Control Hosp Epidemiol 2017;38:266-72.

15. Isendahl J, Giske CG, Hammar U, et al. Temporal dynamics and risk factors for bloodstream infection with extended-spectrum $\beta$-lactamase-producing bacteria in previously colonized individuals: National populationbased cohort study. Clin Infect Dis 2018. [Epub ahead of print].

16. Razazi K, Mekontso Dessap A, Carteaux G, et al. Frequency, associated factors and outcome of multi-drugresistant intensive care unit-acquired pneumonia among patients colonized with extended-spectrum $\beta$-lactamase- 
producing Enterobacteriaceae. Ann Intensive Care 2017;7:61.

17. Bruyère R, Vigneron C, Bador J, et al. Significance of Prior Digestive Colonization With Extended-Spectrum $\beta$-Lactamase-Producing Enterobacteriaceae in Patients With Ventilator-Associated Pneumonia. Crit Care Med 2016;44:699-706.

18. Barbier F, Bailly S, Schwebel C, et al. Infection-related ventilator-associated complications in ICU patients colonised with extended-spectrum $\beta$-lactamase-producing Enterobacteriaceae. Intensive Care Med 2018;44:616-26.

19. Barbier F, Pommier C, Essaied W, et al. Colonization and infection with extended-spectrum $\beta$-lactamase-producing Enterobacteriaceae in ICU patients: what impact on outcomes and carbapenem exposure? J Antimicrob Chemother 2016;71:1088-97.

20. Prinapori R, Guinaud J, Khalil A, et al. Risk associated with a systematic search of extended-spectrum $\beta$-lactamase-producing Enterobacteriaceae. Am J Infect Control 2013;41:259-60.

21. Armand-Lefèvre L, Angebault C, Barbier F, et al. Emergence of imipenem-resistant gram-negative bacilli in intestinal flora of intensive care patients. Antimicrob Agents Chemother 2013; 57:1488-95.

22. Lepper PM, Grusa E, Reichl H, et al. Consumption of imipenem correlates with beta-lactam resistance in Pseudomonas aeruginosa. Antimicrob Agents Chemother 2002;46:2920-5.

23. Pilmis B, Jullien V, Tabah A, et al. Piperacillin-tazobactam as alternative to carbapenems for ICU patients. Ann Intensive Care 2017;7:113.

Cite this article as: Pilmis B, Zahar JR. Ventilator-associated pneumonia related to ESBL-producing gram negative bacilli. Ann Transl Med 2018;6(21):424. doi: 10.21037/atm.2018.09.34
24. Donskey CJ. Antibiotic regimens and intestinal colonization with antibiotic-resistant gram-negative bacilli. Clin Infect Dis 2006;43 Suppl 2:S62-9.

25. Lob SH, Nicolle LE, Hoban DJ, et al. Susceptibility patterns and ESBL rates of Escherichia coli from urinary tract infections in Canada and the United States, SMART 2010-2014. Diagn Microbiol Infect Dis 2016;85:459-65.

26. Roberts JA, Ulldemolins M, Roberts MS, et al. Therapeutic drug monitoring of $\beta$-lactams in critically ill patients: proof of concept. Int J Antimicrob Agents 2010;36:332-9.

27. Vincent JL, Bassetti M, François B, et al. Advances in antibiotic therapy in the critically ill. Crit Care 2016;20:133.

28. Guet-Revillet H, Tomini E, Emirian A, et al. Piperacillin/ tazobactam as an alternative antibiotic therapy to carbapenems in the treatment of urinary tract infections due to extended-spectrum $\beta$-lactamase-producing Enterobacteriaceae: an in silico pharmacokinetic study. Int J Antimicrob Agents 2017;49:62-6.

29. Grall N, Lazarevic V, Gaïa N, et al. Unexpected persistence of extended-spectrum $\beta$-lactamase-producing Enterobacteriaceae in the faecal microbiota of hospitalised patients treated with imipenem. Int J Antimicrob Agents 2017;50:81-7.

30. Gallah S, Benzerara Y, Tankovic J, et al. $\beta$ LACTA test performance for detection of extended-spectrum $\beta$-lactamase-producing Gram-negative bacilli directly on bronchial aspirates samples: a validation study. Clin Microbiol Infect 2018;24:402-8. 